\title{
Particle in the Brusselator model with flow
}

\author{
P.V. Kuptsov ${ }^{\mathrm{a}, *}$, S.P. Kuznetsov ${ }^{\mathrm{a}}$, E. Mosekilde ${ }^{\mathrm{b}}$ \\ ${ }^{a}$ Institute of Radio Engineering and Electronics, Russian Academy of Science, Zelenaya 38, Saratov 410019, Russia \\ ${ }^{\mathrm{b}}$ Department of Physics, The Technical University of Denmark, $2800 \mathrm{Kgs}$. Lyngby, Denmark
}

Received 16 July 2001; received in revised form 16 November 2001; accepted 20 November 2001

Communicated by L. Kramer

\begin{abstract}
We consider the interaction of a small moving particle with a stationary space-periodic pattern in a chemical reactiondiffusion system with a flow. The pattern is produced by a one-dimensional Brusselator model that is perturbed by a constant displacement from the equilibrium state at the inlet. By partially blocking the flow, the particle gives rise to a local increment of the flow rate. For certain parameter values a response with intermittent Hopf and Turing type structures is observed. In other regimes a wave of substitution of missing peaks runs across the pattern. () 2002 Elsevier Science B.V. All rights reserved.
\end{abstract}

PACS: $82.40 . \mathrm{Bj} ;$ 47.20.Ky

Keywords: Reaction-diffusion system; Hopf and Turing instability; Pattern response; Intermittency

\section{Introduction}

Pattern formation is a fundamental problem in a study of far-from-equilibrium phenomena in spatially extended systems. The general idea of such investigations is to search for a solution that a system selects above the point of instability of the homogeneous steady state, and analyze the stability of this solution with respect to all possible perturbations [1]. This approach allows to understand and predict a variety of different phenomena including formation of structures that are similar to the patterns we observe in the living world [2-4].

Reaction-diffusion systems represent a widely studied class of chemical systems that can produce

\footnotetext{
* Corresponding author. Tel.: +7-8452-24-86-85; fax: +7-8452-51-45-40.

E-mail address: kupav@mail.ru (P.V. Kuptsov).
}

complex spatio-temporal phenomena [5,6]. The basic theory of pattern formation in such systems was developed by Turing [7] in his seminal paper "The chemical basis of morphogenesis". In its simplest form, the Turing instability arises from the interaction of two chemical species: an autocatalytic activator and an inhibitor that limits the growth of the activator. A main condition for the Turing instability to arise is that the inhibitor diffuses significantly faster than the activator. This allows the growth of localized activated areas and prevents their spreading in space. If this condition is not satisfied, a Hopf bifurcation (leading to spatially homogeneous temporal oscillations) occurs instead.

Over the years a variety of different models have been developed in order to study the formation of Turing structures $[2,3,8]$, and the possibility of molecular Turing structures in the biochemistry of the cell has been investigated, for instance, by Hasslacher 
et al. [9]. The first experimental observation of Turing structures was made in 1990 by Castets et al. [10,11] using the chlorite-iodide-malonic acid (or so-called CIMA) reaction that in other circumstances is known to produce oscillations and traveling waves.

During the last few years the interest in pattern formation in chemical reaction-diffusion systems has increased since it was suggested $[12,13]$ that stationary patterns could appear for systems with identical diffusion constants, provided the reaction-diffusion processes take place in the presence of a flow that is constantly perturbed at the inlet. The existence of these new type of patterns was verified in experiments by Kærn and Menzinger [14].

In this paper we use a one-dimensional Brusselator model [2] with flow to study how a fully formed pattern is modified by a small particle that travels through the pattern as it is dragged by the flow. For certain parameter values the pattern responds by the appearance of intermittent Hopf- and Turing type oscillations. For other parameters we can observe how a wave of substitution of missing peaks runs across the pattern.

\section{Brusselator model with flow}

The Brusselator represents a well-known example of a reaction-diffusion model. In the presence of a flow, this model takes the form [12]:

$$
\begin{aligned}
& \frac{\partial U}{\partial t}+c \frac{\partial U}{\partial x}=A-(B+1) U+U^{2} V+\sigma \frac{\partial^{2} U}{\partial x^{2}}, \\
& \frac{\partial V}{\partial t}+c \frac{\partial V}{\partial x}=B U-U^{2} V+\frac{\partial^{2} V}{\partial x^{2}} .
\end{aligned}
$$

Here, $U$ and $V$ denote the concentrations of the interacting chemical species, $A$ and $B$ the externally controlled feed concentrations, $c$ the flow rate and $\sigma$ the ratio of the two diffusion constants. In what follows the reagents are assumed to be pumped continuously into the reaction space from the left to leave the reactor to the right. We will assume that the left-boundary concentration is constant,

${\frac{\partial U}{\partial t}{ }_{\mid x=0}}=\frac{\partial V}{\partial t}_{\mid x=0}=0$ and the right-boundary condition is free,

$\frac{\partial U}{\partial x}_{\mid x=L}=\frac{\partial V}{\partial x}_{\mid x=L}=0$,

with $L$ denoting the length of the reactor.

The system (1) obviously allows for the homogeneous steady state solution

$U_{0}=A, \quad V_{0}=\frac{B}{A}$.

If the left-boundary condition is taken to be

$U_{\mid x=0}=U_{0}, \quad V_{\mid x=0}=V_{0}$,

and the control parameters $A, B$ and $\sigma$ are changed, one observes that this solution can lose its stability via two different bifurcations $[2,12]$. The first one, involving a Hopf instability with the threshold point at

$B \equiv B_{\mathrm{H}}=1+A^{2}$,

is associated with the appearance of spatially homogeneous oscillations. The second, involving a Turing instability with the threshold at

$B \equiv B_{\mathrm{T}}=\left(1+A \sigma^{1 / 2}\right)^{2}$,

manifests itself by the growth of a mode with non-zero wave number and with a temporal frequency that is proportional to the flow rate.

The Hopf and Turing instabilities in the system (1) may be absolute or convective [12]. When the flow rate is small, a localized initial perturbation gives rise to growing amplitudes at all points in space and the instability is absolute. With large flow rates, on the other hand, the instability becomes convective. The growing perturbation drifts in such a way that observation at any fixed point in the laboratory frame asymptotically shows an amplitude that decays towards zero.

To study the model equation (1) via numerical simulation we have applied a Crank-Nicholson semiimplicit scheme that retains the second order of the local approximation [15]. If nothing else is explicitly stated, the values of the time and space steps are selected as $\Delta x=\Delta t=0.1$.

Fig. 1 (redrawn from [12]) shows three parameter planes $(c, B)$ of the system (1) corresponding to different values of parameter $A$ at fixed $\sigma$. In all the 
(a)

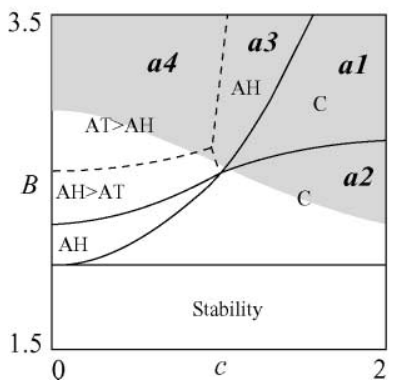

(b)

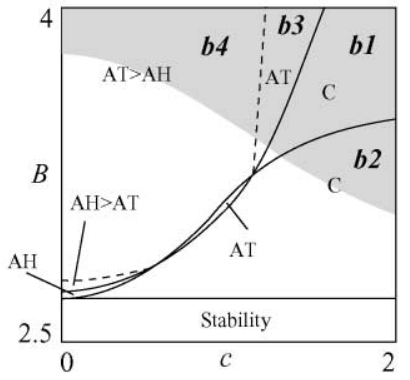

(c)

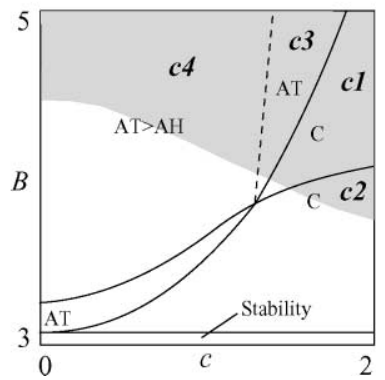

Fig. 1. Sections of the parameter space for system (1) with $\sigma=0.25$ [12]: (a) $A=1$; (b) $A=1.3$; (c) $A=1.5$. Solid curves correspond to zero growth rate for the Hopf and Turing modes, while the two modes have the same growth rates along the dashed curves. Different types of behavior are denoted by the letters A (absolute instability) and C (convective instability), H (Hopf mode) and T (Turing mode). Sign ">" indicates what mode has the larger growth rate. Shading marks the parameter regions where the edge perturbation becomes undamped in space. $a 1-c 4$ denote different domains in which the behavior of the system is considered.

diagrams we observe the domain of stability, located below the threshold line $B=$ constant that is defined by Eq. (7) or Eq. (8). Above these thresholds there are domains denoted with letter $\mathrm{C}$ where Hopf and Turing modes are both convectively unstable. Then, we observe domains with absolutely unstable Hopf (AH) or Turing (AT) modes. And finally, for large values of $B$, we have the domains where Hopf and Turing modes are both absolutely unstable. Here the sign " $>$ " indicates the mode with the larger growth rate.

Fig. 2 presents a typical spatio-temporal behavior of system (1) when Hopf and Turing modes are both convectively unstable. One sees how the two types of instability develop in the system simultaneously and compete with each other. The Hopf areas are identified by large scale inhomogeneities with relatively large time period and little variation in space. The Turing areas involve high-frequency oscillations in time and space. The time oscillations are proportional to the flow rate and are absent for an observer that moves with the flow as indicated with the black straight line in the figure.

When the Hopf and Turing modes are both unstable, the development of Hopf or Turing areas in the different space regions of the system depends on the initial perturbation of the steady state (5). Turing areas develop from perturbations that change rapidly in space, while the seeds of the Hopf areas are the extended (a)

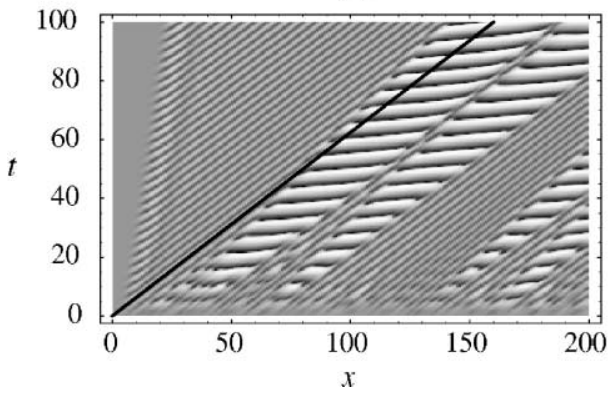

(b)

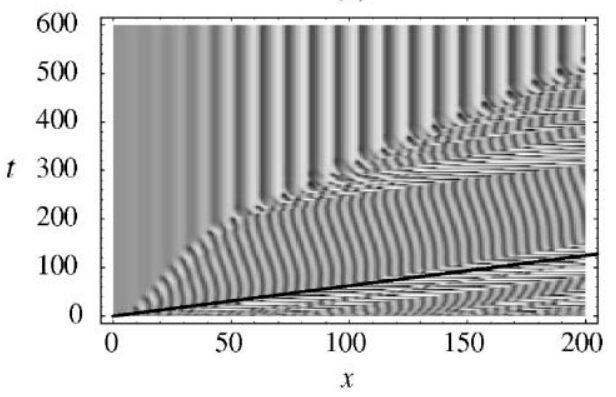

Fig. 2. Examples of spatio-temporal behavior of the system (1) for the case $b 1$ (see Table 1): (a) Convective instability. Both the Hopf (time oscillations with a large period) and Turing (fast space-time oscillations) modes develop and compete with each other. (b) Stationary in time and spatially undamped periodic pattern, forming for constant edge perturbation $\epsilon_{U}=0.01$ (see (9)). Gray levels represent the concentration of $V$, with the lighter levels indicating higher concentrations. The sloping black lines represent the flow rate. 
homogeneous deviations from the steady state. Because of this difference in excitation mechanisms, the relative growth rates of the Hopf and Turing modes depend on the spatial step size of the numerical simulations. For a smaller step the system becomes more unstable in the Turing sense because local approximation errors appear on the smaller space scales and hence take part mainly in excitation of a Turing mode. Hence we are not allowed to decrease the step of the numerical simulations as much as possible trying to get the best result because we can give an artificial advantage to one of the modes and lose some effects of competition.

When a constant edge perturbation is applied at the inlet of the system,

$U_{\mid x=0}=U_{0}+\epsilon_{U}, \quad V_{\mid x=0}=V_{0}\left(\epsilon_{U} \neq 0\right)$,

stationary space-periodic patterns arise [12,13]. In Fig. 1 the white domains correspond to situations where the patterns decay in space, and shading marks the regions where they are spatially undamped. An example of an undamped pattern is presented in Fig. 2(b). These patterns will be the subject of our interest in what follows.

\section{Perturbation of the fully developed pattern}

Let us suppose that the pattern is fully developed in system (1) and that one drops a particle into the flow at the reactor inlet. This perturbation is assumed to be small enough so that it can not only destroy the pattern and switch the system to a different solution, but we also expect to observe some response from the pattern.

We suppose that the reactor is a thin tube, and the particle size $p$ is much smaller than the reactor length. The particle partly blocks the flow and then produces a local increment $c_{p}$ of the flow rate. The flow drags the particle, but because of friction with the reactor walls the speed of the particle $v$ is less than the flow rate, $v<c$. Neglecting transient processes around the particle one can write the following equations:

$$
\begin{aligned}
& \frac{\partial U}{\partial t}+\left(c+c_{p} F_{p}(x-v t)\right) \frac{\partial U}{\partial x} \\
& =A-(B+1) U+U^{2} V+\sigma \frac{\partial^{2} U}{\partial x^{2}},
\end{aligned}
$$

$$
\begin{aligned}
& \frac{\partial V}{\partial t}+\left(c+c_{p} F_{p}(x-v t)\right) \frac{\partial V}{\partial x} \\
& =B U-U^{2} V+\frac{\partial^{2} V}{\partial x^{2}},
\end{aligned}
$$

$F_{p}(x)= \begin{cases}1 & \text { if } 0 \leq x \leq p, \\ 0 & \text { otherwise }\end{cases}$

The response of the pattern depends on the domain in parameter space where the system is operating. The shaded regions in Fig. 1 are each divided into four subdomains, that are denoted as $a 1-c 4$. Let us fix some points in these subdomains and consider the behavior of the system in each of these points. The parameter values in the points are collected in Table 1 . The corresponding spatio-temporal diagrams are depicted in Figs. 3-6. These figures are obtained for a fixed particle size $p=0.1$ that is equal to the numerical simulation step.

Inspection of the figures shows that the most intensive interaction takes place near the reactor inlet. The response of the pattern that arises here is carried by the flow and enlarges to the left and right. It is composed of areas of two types. The Hopf type areas involve slow time oscillations, and the Turing areas have fast space-time oscillations. When the particle is far from the reactor inlet the pattern is rather insensitive to the

\begin{tabular}{|c|c|c|c|c|c|c|c|c|c|c|c|c|}
\hline & $a 1$ & $a 2$ & $a 3$ & $a 4$ & $b 1$ & $b 2$ & $b 3$ & $b 4$ & $c 1$ & $c 2$ & $c 3$ & $c 4$ \\
\hline$A$ & 1.0 & & & & 1.3 & & & & 1.5 & & & \\
\hline$B$ & 2.86 & 2.46 & 3.10 & 2.99 & 3.55 & 3.29 & 3.62 & 3.60 & 4.23 & 3.90 & 4.35 & 4.25 \\
\hline$c$ & 1.69 & 1.74 & 1.15 & 0.67 & 1.60 & 1.63 & 1.26 & 0.92 & 1.79 & 1.84 & 1.53 & 1.10 \\
\hline
\end{tabular}
particle.

Table 1

Values of parameters that are used to illustrate the behavior of system (1) in the different domains of Fig. 1 (for all cases $\sigma=0.25$ ) 
(a)

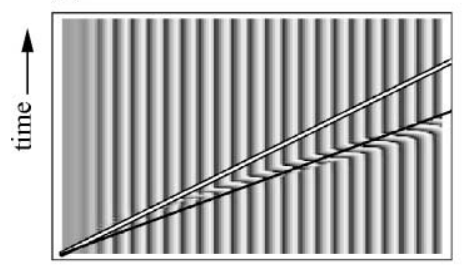

(b)

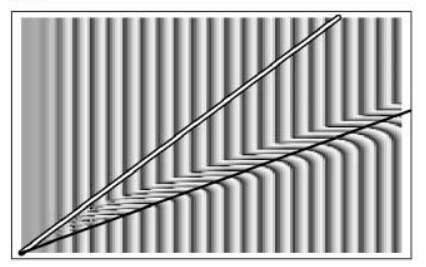

space $\longrightarrow$ (c)

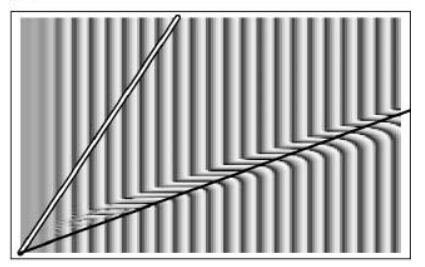

Fig. 3. Particle in a Brusselator model with flow for the case $a 1$ : (a) $v=0.75 c, c_{p}=0.015 c$; (b) $v=0.5 c, c_{p}=0.05 c$; (c) $v=0.25 c$, $c_{p}=10 c$. The particle size is $p=0.1$ (i.e. one step of the numerical simulation). The reactor length is 300 and the watching time is 300 . The stationary pattern is obtained for $\epsilon_{U}=0.01$. White sloping lines are the trajectories of the particle, and the black lines indicate the flow rate. The response is of Hopf type. For larger values of $c_{p}$ one observes a secondary response that looks like "ripples on the water". Note that the response area is very regular.

(a)

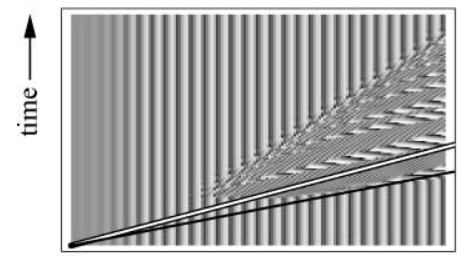

(b)

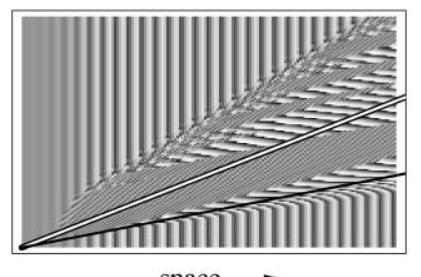

(c)

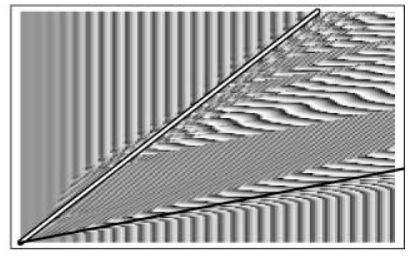

Fig. 4. Similar pictures as in Fig. 3, but for the case $b 1$ : (a) $v=0.75 c, c_{p}=10^{-15} c$; (b) $v=0.5 c, c_{p}=10^{-5} c$; (c) $v=0.25 c, c_{p}=10 c$. The watching time is 600. The response area is irregular and its appearance is fairly insensitive to the particle perturbation $c_{p}$ and rate $v$. It looks the same for different values of $v$ and changes very slowly with $c_{p}$. Note how Turing type regions are intermittent with tongues of Hopf type.

\subsection{Case al}

As seen from Fig. 3 the whole response area is of Hopf type. With large $c_{p}$ the main area is followed by a secondary response that looks like "ripples on the water".
This case is perhaps the most interesting among all the considered cases, because of the good "consensus" between the response and the pattern. As a result of the interaction with the particle, several peaks of the pattern disappear and a wave of substitution of absent peaks runs across the pattern. Depending on (a)

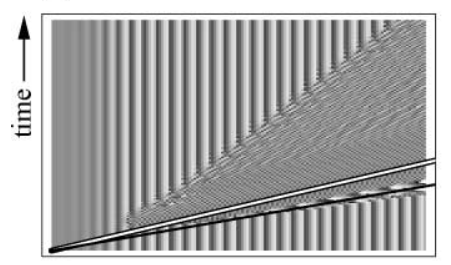

(b)

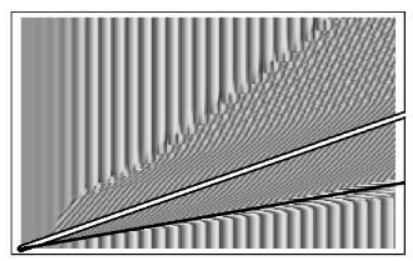

space (c)

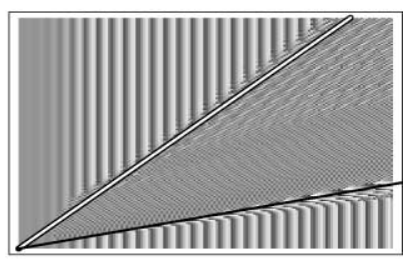

Fig. 5. The same as in Fig. 3 for the case $c 1$ : (a) $v=0.75 c, c_{p}=10^{-15} c$; (b) $v=0.5 c, c_{p}=10^{-5} c$; (c) $v=0.25 c, c_{p}=10 c$. The watching time is 600 . The response area is of Turing type. Note that the response looks the same for very different values of $v$ and $c_{p}$. 
(a)

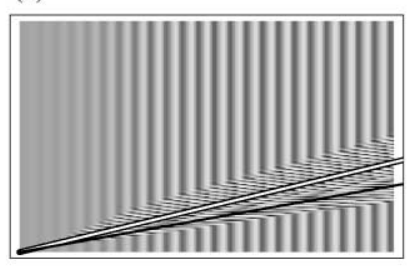

(d)

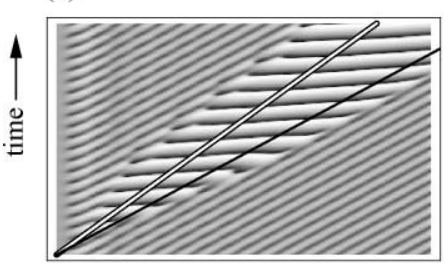

(g)

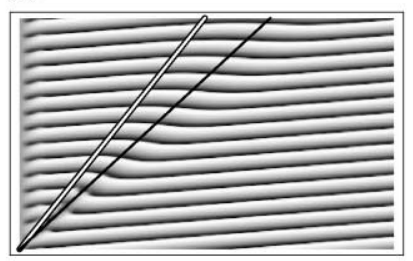

(b)

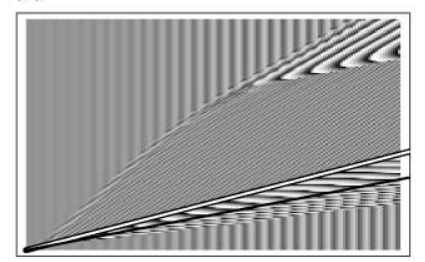

(e)

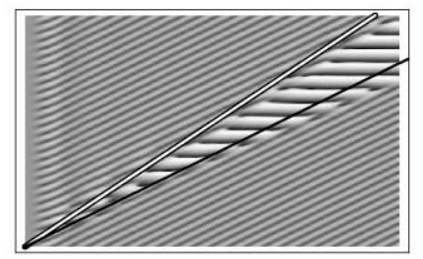

(h)

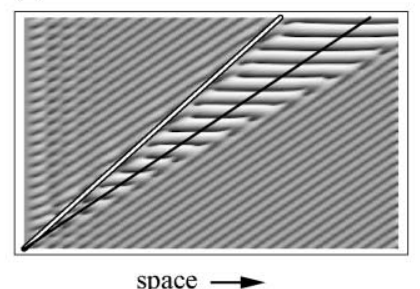

(c)

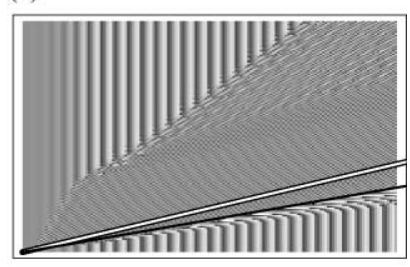

(f)

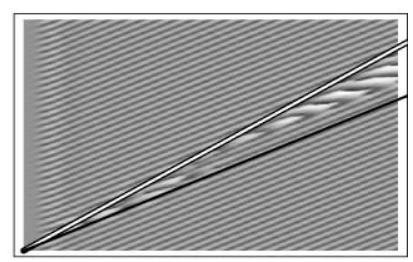

(i)

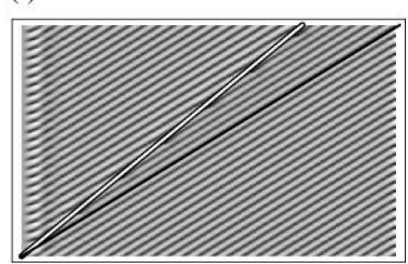

Fig. 6. The particle flight for cases $a 2-c 4$ at $v=0.75 c, c_{p}=10 c$ : (a) case $a 2$; (b) case $b 2$; (c) case $c 2$; (d) case $a 3$; (e) case $b 3$; (f) case $c 3$; (g) case $a 4$; (h) case $b 4$; (i) case $c 4$. For cases $a 2-c 2$ the reactor length is 300 , and the watching time is 600 . For the other cases the space-time extensions are $100 \times 100$. For $a$-cases the response is mainly of Hopf type and for $c$-cases it is of Turing type. Note that cases $a 2, b 2$ and $c 2$ are most similar to the previously represented cases $a 1, b 1$ and $c 1$.

the parameters of the particle $c_{p}$ and $v$, one can see the wave of weak peak oscillations, the substitutions of one peak, the substitutions of two peaks, and so on.

The observed phenomenon may be interpreted as a chemical analog-digital conversion. The parameters of the particle may be considered as an input signal and the number of disappeared peaks as an output signal.

\subsection{Case bl}

Fig. 4 illustrates this case. The response is seen to be less regular than in the previous case. One can observe a Turing type response which is intermittent with tongues of Hopf type.

In the case under consideration the sensitivity of the pattern is much higher than in the previous case. A significant response arises even at very small values of $c_{p}$. The appearance of the response area depends rather weakly on $c_{p}$ and $v$. Increase of $c_{p}$ leads mainly to a displacement of the response area towards the lower-left corner of the spatially temporal diagram, indicating that the response grows faster in both time and space.

\subsection{Case cl}

As one can see from Fig. 5, the response is of Turing type. As in the previous case, the sensitivity of the pattern is high, and the appearance of the response area depends weakly on $c_{p}$ and $v$.

Some of the other cases are illustrated in Fig. 6. In $a$-cases we observe a Hopf type response while in $c$-cases a Turing type of response is realized more often. Note that the cases $a 2, b 2$ and $c 2$ are rather similar to the previously discussed cases. For $b 2$ 
the Turing type response is intermittent with Hopf tongues. The only difference is the absence of the "consensus" between the pattern and the response in case $a 2$.

\section{Intermittent Turing-Hopf response}

Let us discuss the represented diagrams of responses from a different point of view. We can consider the cases $a 1, b 1$ and $c 1$ to represent three steps along some curve in parameter space. In other words we introduce a new control parameter, which is a function of $A, B$, $c$ and $\sigma$. When increasing this parameter, one first observes a response of the Hopf type. Then the response area is of Turing type with Hopf tongues. Finally, the response becomes Turing type. We can interpret this scenario as a transition from the Hopf to the Turing response through some kind of intermittency. Fig. 7 illustrates this in more detail. Here, the oscillations of $V$ are shown at the fixed point $x$. In diagram (a) one can see the low-frequency oscillation corresponding to a Hopf type response. In diagram (b) the lowfrequency windows are interrupted with the highfrequency areas of the Turing type. And finally, in diagram (c) there are high-frequency oscillations which correspond to a Turing type response.

To characterize the observed intermittency let us consider the time series obtained at the fixed point $x$ for the case $b 1$, and build the distribution of lengths of windows of Hopf oscillations. Because the considered model is fully deterministic and does not contain any noise, we obtain the same time series for the same initial and boundary conditions. That is of course not the case for a real system, and to avoid this problem we produce a number of the numerical calculations with slightly different time steps. The Hopf windows can be easily extracted from the resulting
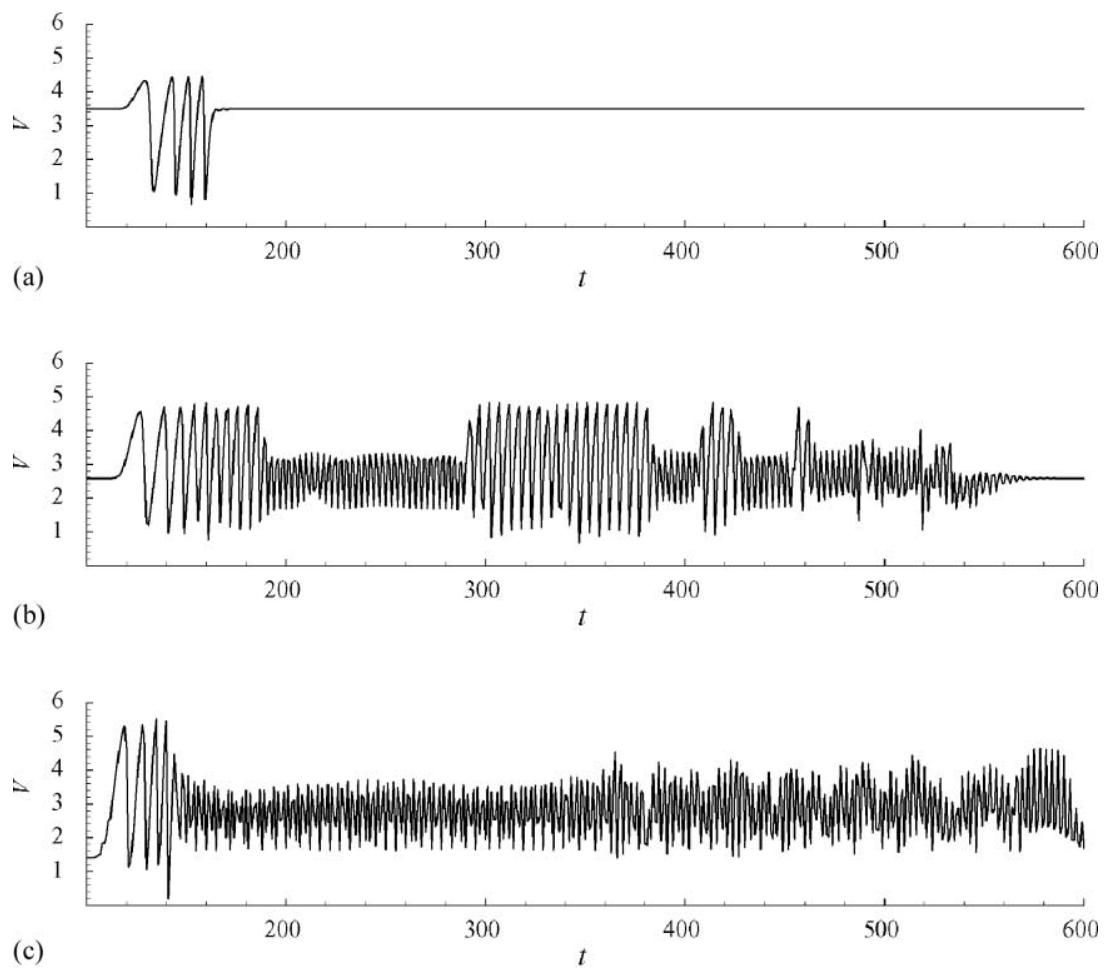

Fig. 7. Temporal variation of $V$ at the point $x=250$ : (a) case $a 1$; (b) case $b 1$; (c) case $c 1$. The plots are drawn at the parameter values corresponding to diagrams (b) of Figs. 3-5. 

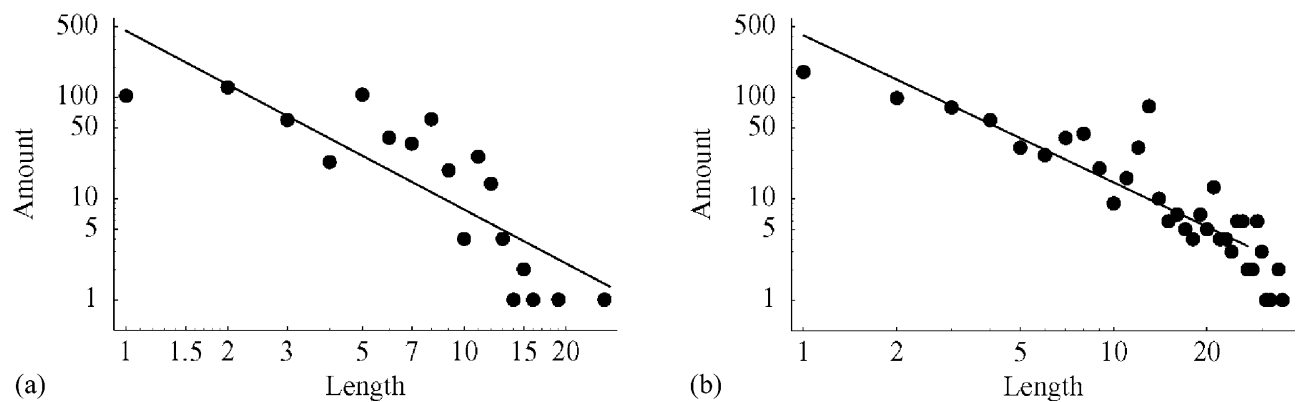

Fig. 8. Distributions of Hopf windows in the time series obtained for Fig. 4(b) at fixed values of $x$. To collect statistics, 100 time series are calculated numerically with random time steps from the interval $0.09<\Delta t<0.11$. The distributions are sketched with dots and double-logarithmic scales are used. Note that a linear approximation is allowed, indicating that the distributions are close to a power law dependence. The slopes of the approximating lines are: (a) $-1.77(x=150)$ and (b) $-1.45(x=290)$.

time series, because of their relatively large amplitude, as seen from Fig. 7. As a measure of window length we use the number of maxima, contained in the window.

In Fig. 8(a) and (b) two distributions are represented for different values of $x$. The diagrams of this figure correspond to Fig. 4(b). One sees that the distributions allow a linear approximation in double-logarithmic scales. This corresponds to a power law dependence in the original linear axes. One sees that far from the system inlet the power law exponent decreases. This can be explained by inspection of Fig. 4. One sees that the Hopf tongues enlarge with $x$ and occupy more and more room for the response area. Hence the amount of large windows increases in the time series and this leads to a reduction of the power law exponent.

The observed structure of the response areas can be explained if one considers also the behavior of system (1) without the particle and edge perturbation,

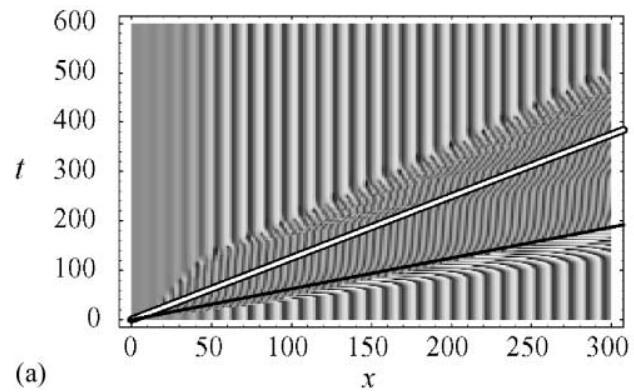

i.e. with $\epsilon_{U}=0$. The pattern is destructed inside the response area, and a transient process takes place. Hence, the instability in the system (1) without the pattern $\left(\epsilon_{U}=0\right)$ develops in the same way as the response area. We illustrate this for the case $b 1$. The responses in Fig. 4 and the spatio-temporal diagram Fig. 2(a) have the same structure. The Turing region is interleaved with Hopf tongues.

Let us finally discuss the dependence of the response on the particle size $p$. As it was mentioned above, to excite the Hopf mode in the system (1), one needs to apply a uniform initial perturbation of large size. Hence, the response area becomes more stable in the Hopf sense for the smaller particle. This property plays an important role in case $b 1$, as illustrated in Fig. 9. This figure is obtained with space and time steps $\Delta x=\Delta t=0.025$. We observe that a small particle cannot excite the Hopf tongues, and the tongues appear if the particle size becomes larger.

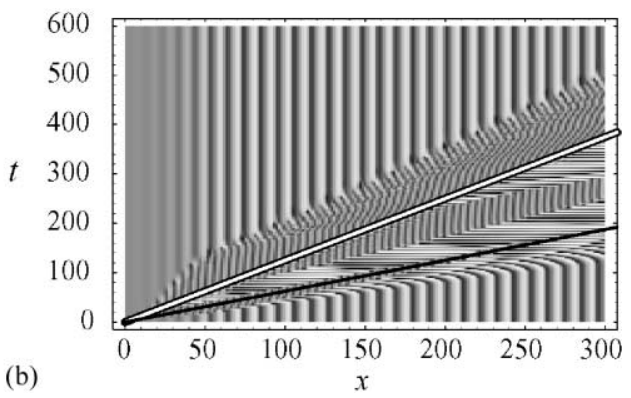

Fig. 9. Pattern response in the case $b 1$ for the different particle sizes $p$ : (a) $p=0.25$; (b) $p=0.5$. The steps in time and space in the numerical simulations are $\Delta x=\Delta t=0.025, v=0.5 c, c_{p}=10 c$. Note how Hopf tongues disappear for the small particle. 


\section{Conclusion}

We have shown several examples of perturbation of the stationary pattern in the Brusselator model with flow in the presence of a small moving particle. The most intense interaction of the particle with the pattern is observed near the system inlet, and far from the inlet the pattern is rather insensitive to the particle. The perturbation region excited by the particle moves downstream and enlarges in space. The pattern is destroyed inside this region and it recreates itself beyond.

We interpret the presence of the perturbed region as a pattern response to the particle moving. Analyzing the oscillations inside the perturbed region, one can detect two kinds of pattern response: Hopf and Turing type. The type of the response depends on the domain in the parameter space where the system is operating. There are domains where the response is pure (Hopf or Turing type) and there are boundary domains with a mixed response. The area of mixed response consists of interleaving tongues of the different types that looks like some form of intermittency. We conclude that it is possible to find curves in parameter space so that when moving along such a curve one can observe a transition from the Hopf type response to the Turing type response through intermittency. The appearance of intermittency depends on the particle size. For a very small particle intermittency is not observed.

In some domain of the parameter space we have found an interesting phenomenon. As a result of interaction with the particle, several peaks of the periodic pattern disappear and a wave of substitution of absent peaks runs across the pattern. The number of missing peaks depends on the particle parameters. This behavior may be interpreted as a chemical analog-digital conversion with the particle parameters as an input signal and the number of absent peaks as the output.

We expect that the reported behavior of the stationary pattern that is perturbed by the moving particle will be significant both for further investigations and different applications. In real experiments and industrial processes it is very difficult to create quite pure mixture of the chemical species. Our study shows how small localized pollutions can distort the fully formed patterns of concentrations. For some parameter values even very small pollution that produces very weak local perturbation of the surrounding media can cause large distortion.

\section{Acknowledgements}

PVK acknowledges support from CRDF (grant REC-006). SPK acknowledges support from RFBR (grant 00-02-17509).

\section{References}

[1] M.C. Cross, P.C. Hohenberg, Pattern formation outside of equilibrium, Rev. Mod. Phys. 65 (1993) 852-1112.

[2] G. Nicolis, I. Prigogine, Self-organization in Nonequilibrium Systems, Wiley, New York, 1977.

[3] J.D. Murray, Mathematical Biology, Springer, New York, 1993.

[4] H. Meinhardt, Models of Biological Pattern Formation, Academic Press, New York, 1982.

[5] B.P. Belousov, A periodic reaction and its mechanism, in: R.J. Field, M. Burger (Eds.), Oscillations and Traveling Waves in a Chemical System, Wiley, New York, 1985, pp. 605613.

[6] A.M. Zhabotinskii, Periodic processes of the oxidation of malonic acid in solution (study of the kinetics of Belousov's reaction), Biofizika 9 (1964) 306-311.

[7] A.M. Turing, The chemical basis of morphogenesis, Philos. Trans. R. Soc. B 237 (1952) 37-72.

[8] F. Baras, J.E. Pearson, M.M. Mansor, Microscopic simulation of chemical oscillations in homogeneous systems, J. Chem. Phys. 93 (1990) 5747-5750.

[9] B. Hasslacher, R. Kapral, A. Lawniczak, Molecular Turing structures in the biochemistry of the cell, Chaos 3 (1993) $7-13$.

[10] V. Castets, E. Dulos, J. Boissonade, P. De Kepper, Experimental evidence of a sustained standing Turing-type nonequilibrium chemical pattern, Phys. Rev. Lett. 64 (1990) 2953-2956.

[11] Q. Ouyang, H. Swinney, Transition to chemical turbulence, Chaos 1 (1991) 411-420.

[12] S.P. Kuznetsov, E. Mosekilde, G. Dewel, P. Borckmans, Absolute and convective instabilities in a one-dimensional Brusselator flow model, J. Chem. Phys. 106 (1997) 76097616.

[13] P. Andreseń, M. Bache, G. Dewel, P. Borckmans, E. Mosekilde, Stationary space periodic structures with equal diffusion coefficients, Phys. Rev. E 60 (1999) 297-301.

[14] M. Kærn, M. Menzinger, Flow-distributed oscillations: stationary chemical waves in a reacting flow, Phys. Rev. E 60 (1999) R3471-R3474.

[15] W.H. Press, S.A. Teukolsky, W.T. Vettering, B.P. Flannery, Numerical Recipes in C, Cambridge University Press, Cambridge, 1992. 